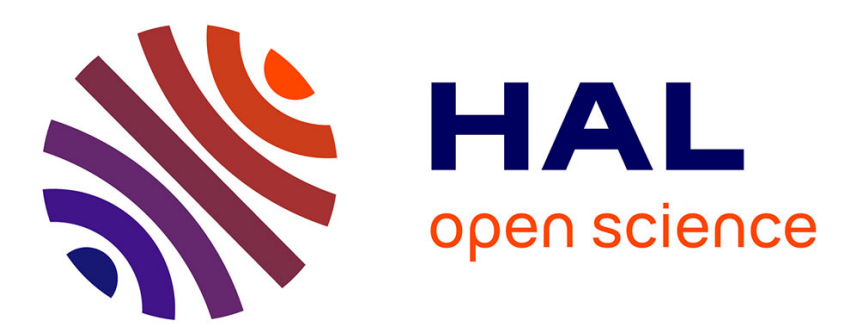

\title{
Numerical modelling of the effective conductivities of composites with arbitrarily shaped inclusions and highly conducting interface
}

\author{
Julien Yvonnet, Qi-Chang He, C. Toulemonde
}

\section{To cite this version: \\ Julien Yvonnet, Qi-Chang He, C. Toulemonde. Numerical modelling of the effective conductivities of composites with arbitrarily shaped inclusions and highly conducting interface. Composites Science and Technology, 2008, 68 (-), pp.2828-2825. 10.1016/j.compscitech.2008.06.008 . hal-00692237}

\section{HAL Id: hal-00692237 \\ https://hal.science/hal-00692237}

Submitted on 7 Sep 2014

HAL is a multi-disciplinary open access archive for the deposit and dissemination of scientific research documents, whether they are published or not. The documents may come from teaching and research institutions in France or abroad, or from public or private research centers.
L'archive ouverte pluridisciplinaire HAL, est destinée au dépôt et à la diffusion de documents scientifiques de niveau recherche, publiés ou non, émanant des établissements d'enseignement et de recherche français ou étrangers, des laboratoires publics ou privés. 


\title{
Numerical modelling of the effective conductivities of composites with arbitrarily shaped inclusions and highly conducting interface
}

\author{
J. Yvonnet ${ }^{\mathrm{a}, *}$, Q.-C. He ${ }^{\mathrm{a}}$ \\ ${ }^{a}$ Université Paris-Est, Laboratoire Modélisation et Simulation Multi Echelle, \\ MSME FRE3160 CNRS, 5 Bd Descartes, 77454 Marne-la-Vallée, France \\ C. Toulemonde ${ }^{\mathrm{b}}$ \\ ${ }^{\mathrm{b}}$ EDF RED - Département MMC \\ Site des Renardières - Avenue des Renardières - Ecuelles, 77818 Moret sur Loing \\ Cedex, France
}

\begin{abstract}
The particulate composite under consideration consists of arbitrarily shaped micro inclusions embedded in a matrix. The interface between the matrix and each inclusion is imperfect and highly conducting, and corresponds to the case of inclusions coated with a highly conducting material. More precisely, the temperature across the interface is continuous while the normal heat flux across the interface suffers a jump related to the surface flux. The purpose of the present work is to elaborate an efficient numerical tool for computing the effective conductivities of the composite by combining the level-set method and the extended finite element method (XFEM). The elaborated numerical procedure has the advantage of avoiding curvilinear coordinates and surface elements in treating imperfect interfaces. It is first validated with the help of some analytical exact and approximate results as benchmarks. It is then applied to determine the inclusion size and shape effects on the effective thermal conductivities of composites with highly conducting coated inclusions and arbitrary geometries. The elaborated numerical procedure is directly applicable to other physically analogous phenomena, such as electrical conduction, dielectrics and diffusion, and to the mathematically identical phenomenon of anti-plane elasticity.
\end{abstract}

Key words: A. Particles-reinforced composites, B. Interface, B. Thermal properties, Extended Finite Element Method 


\section{Introduction}

Effective transport properties of a composite can be significantly affected by the presence of imperfect interfaces between its constituents, as shown experimentally by Garret and Rosenberg [9] and Hasselman and Donaldson [11]. In the context of thermal conduction, two imperfect interface models have been widely studied and used in the literature. The first one is the well-known Kapitza's thermal resistance model, according to which the temperature is discontinuous across an interface and the normal heat flux component is continuous and proportional to the temperature jump across the interface. The second one is the highly conducting interface model, according to which the temperature is continuous across an interface but the normal heat flux component suffers a jump which must verify the Young-Laplace equation. The Kapitza's thermal resistive interface model (or the highly conduction interface model) can be rigorously derived in an asymptotic way from the physicallybased configuration where a very thin lowly (or highly) conducting interphase is situated between two media $([22,21,19,10,4])$.

The present work is concerned with the problem of determining the effective thermal conductivities of a particulate composite in which the interface between each inclusion and the matrix can be suitably described by the highly conducting (HC) interface model. This problem has been studied by Torquato and Rintoul [24], Cheng and Torquato [5], Lipton [16], Miloh and Benveniste [19], Lipton and Talbot [17], Duan and Karihaloo [6] and Le Quang et al. [15], among others. These works have been focused on the derivation of analytical exact or approximate closed-form results and limited to the case where inclusions are of uniform size and simple geometrical shape. Quite different from the previous ones, the present work has the purpose of elaborating an efficient numerical procedure for computing the effective thermal conductivities of a particulate composite in which the inclusions have different sizes and arbitrary shapes and the interfaces are highly conducting. To achieve this purpose, we shall first formulate the problem in the weak form and then solve it by combining the level-set method (LSM) and extended finite element method (XFEM).

From the numerical point of view, using both LSM and XFEM to deal with imperfect interfaces such as the HC interface presents two important advantages. First, since an interface is described as a level set of a function defined in an Euclidean space, no curvilinear coordinates are needed. Second, since the discontinuities across an interface are modeled by enriching the relevant finite element fields with appropriate discontinuous functions, a regular mesh-

\footnotetext{
* Correspondance to J. Yvonnet

Email address: julien.yvonnet@univ-paris-est.fr (J. Yvonnet).
} 
ing suffices and no surface elements are required. For more details on XFEM and its combination with LSM, we refer to $[3,2,20,23]$. The present work can be viewed as a continuation of our previous one [25] where a numerical approach was proposed to compute the effective elastic properties of multiphase materials with elastic coherent interfaces. However, that work was limited to the two-dimensional (2D) elasticity whereas the present one concerns the three-dimensional (3D) thermal conduction.

The elastic counterpart of the HC interface model is the interface stress model (see, e.g., Gurtin and Murdoch [8]; Benveniste [4]; Yvonnet et al. [25]). According to the latter, the displacement vector is continuous across an interface, the traction vector across the interface is discontinuous and verifies the corresponding Young-Laplace equation, and the interface stress tensor is related to the interface strain tensor by the two-dimensional Hooke law. It is known (see, e.g., [18]; [13]) that the interface stress model is suitable for characterizing the elastic effects of interfaces/surfaces in nano-structured materials. However, the thermal effects of interfaces or surfaces in these materials seem to be much more complex (see, e.g., [14]; [1]), so that they could not be described by the HC interface model. Therefore, the numerical procedure proposed in the present paper is well suited for composites with micro-inclusions but not for nano-inclusions.

The paper is organised as follows. In the next section, the physical setting and local governing equations of the problem under investigation are specified and the weak form suitable for a numerical treatment of the problem is established. In section 3 , how to numerically solve the problem by employing and implementing LSM and XFEM is detailed. In section 4, the numerical procedure elaborated in section 3 is first tested and validated with the help of some analytical exact and approximate results as benchmarks. It is then applied to studying the inclusion size and shape effects on the effective thermal conductivities of particulate composites with inclusions coated by highly conducting material. A few concluding remarks are drawn in the last section.

\section{Physical setting and mathematical formulation of the problem}

\subsection{Local constitutive laws and balance equations}

The particulate composite under investigation consists of a matrix in which micro-sized inclusions are embedded. Let $\Omega$ be the domain occupied by a representative volume element (RVE) of the composite in a three-dimensional (3D) Euclidean space $\mathbb{R}^{3}$ and let $\partial \Omega$ be the boundary of $\Omega$. The subdomain of $\Omega$ associated to the matrix is denoted by $\Omega^{(2)}$, the subdomains of $\Omega$ corresponding 


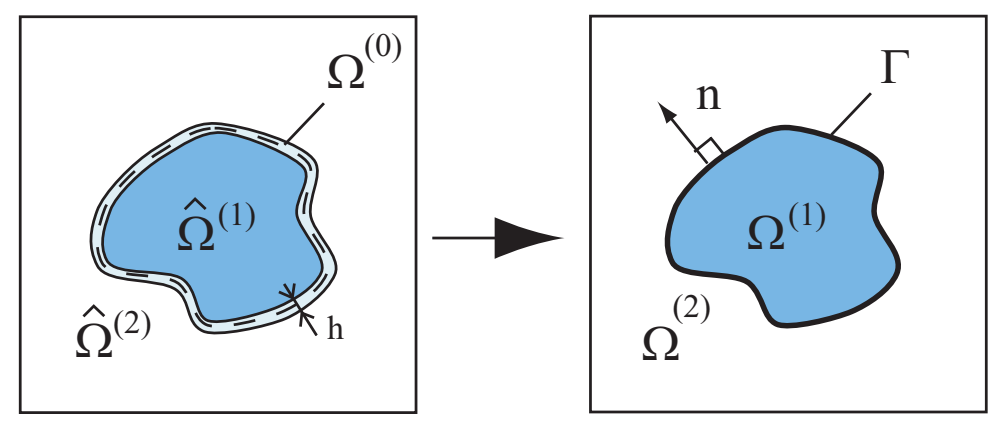

(a)

(b)

Fig. 1. Replacement of an interphase by an imperfect interface; (a) three-phase problem; (b) two-phase problem with imperfect transmission conditions.

to the inclusions are collectively designated by $\Omega^{(1)}$, and the interface between $\Omega^{(1)}$ and $\Omega^{(2)}$ is inside $\partial \Omega$ and symbolized by $\Gamma$.

The materials forming the matrix and inclusions of the composite are assumed to be individually homogeneous and the interface between the matrix and inclusions is taken to be imperfect and more precisely to be HC. We denote by $T^{(i)}$ and $\mathbf{q}^{(i)}$ the temperature and heat flux vector fields respectively over $\Omega^{(i)}(i=1,2)$. Since the interface $\Gamma$ is $\mathrm{HC}$ (see, e.g., [19]), the temperature field is continuous across $\Gamma$, i.e.,

$$
\llbracket T \rrbracket=T^{(2)}-T^{(1)}=0 \text { on } \Gamma,
$$

but the heat flux vector jumps across $\Gamma$, i.e.,

$$
\llbracket \mathbf{q} \rrbracket \cdot \mathbf{n}=\left(\mathbf{q}^{(2)}-\mathbf{q}^{(1)}\right) \cdot \mathbf{n} \neq 0 \text { on } \Gamma \text {. }
$$

Above and hereafter, the jump of any quantity $\bullet$ across $\Gamma$ is defined by $\llbracket \bullet \rrbracket=$ -(2) $\bullet^{(1)}$ and $\mathbf{n}$ represents the unit vector normal to $\Gamma$ and directed from $\Omega^{(1)}$ into $\Omega^{(2)}$.

For later use and without loss of generality, let the interface $\Gamma$ be characterized by a function $\phi: \mathbb{R}^{3} \rightarrow \mathbb{R}$ as its zero-level set:

$$
\Gamma=\left\{\mathbf{x} \in \mathbb{R}^{3} \mid \phi(\mathbf{x})=0\right\} .
$$

The unit vector normal to $\Gamma$ is then calculated via the gradient $\nabla \phi$ of $\phi$ by

$$
\mathbf{n}(\mathbf{x})=\frac{\nabla \phi(\mathbf{x})}{\|\phi(\mathbf{x})\|}
$$

provided $\nabla \phi(\mathbf{x}) \neq \mathbf{0}$ for every $\mathbf{x} \in \Gamma$. Next, we introduce two complementary orthogonal projection operators $\mathbf{P}^{\perp}(\mathbf{x})$ and $\mathbf{P}(\mathbf{x})$ by

$$
\mathbf{P}(\mathbf{x})=\mathbf{I}-\mathbf{P}^{\perp}(\mathbf{x})=\mathbf{I}-\mathbf{n}(\mathbf{x}) \otimes \mathbf{n}(\mathbf{x})
$$


where $\mathbf{I}$ is the second-order identity tensor. Geometrically, $\mathbf{P}^{\perp}(\mathbf{x})$ corresponds to the projection along the normal to $\Gamma$ at $\mathbf{x}$, and $\mathbf{P}(\mathbf{x})$ to the projection on the plane tangent to $\Gamma$ at $\mathbf{x}$.

Within the framework of steady thermal conduction and in the absence of heat source, the bulk energy conservation equation reads

$$
\operatorname{div} \mathbf{q}^{(i)}=0 \text { in } \Omega^{(i)}
$$

and the interface energy conservation equation takes the form

$$
-\operatorname{div}_{s} \mathbf{q}_{s}=\left(\mathbf{q}^{(2)}-\mathbf{q}^{(1)}\right) \cdot \mathbf{n} \text { on } \Gamma
$$

where $\mathbf{q}_{s}$ is the interface heat flux vector field defined on $\Gamma$ and tangent to $\Gamma$. Equation (7) is the well-known Young-Laplace one. In $(7), \operatorname{div}_{s}(\bullet)$ is the surface divergence operator given by

$$
\operatorname{div}_{s} \mathbf{w}=\operatorname{tr}(\mathbf{P} \nabla \mathbf{w})=\mathbf{P}: \nabla \mathbf{w}
$$

for any differentiable vector field $\mathbf{w}$ defined on $\Gamma$.

The materials forming the matrix and inclusions are assumed to comply with the Fourier law. Thus, the local thermal conduction law reads

$$
\mathbf{q}^{(i)}(\mathbf{x})=-\mathbf{K}^{(i)} \nabla T^{(i)}(\mathbf{x}) \text { in } \Omega^{(i)}
$$

where $\mathbf{K}^{(i)}$ is the thermal conductivity tensor of the material constituting the matrix $(i=2)$ or the inclusions $(i=1)$. In addition, we make the assumption that the thermal conduction of the imperfect interface $\Gamma$ is also governed by the Fourier law:

$$
\mathbf{q}_{s}(\mathbf{x})=-\mathbf{K}_{s} \nabla_{s} T(\mathbf{x}) \text { on } \Gamma .
$$

In this equation, $\mathbf{K}_{s}$ is the interface thermal conductivity tensor of $\Gamma$ having the property that $\mathbf{K}_{s}=\mathbf{P} \mathbf{K}_{s} \mathbf{P}$ and $\nabla_{s}(\bullet)$ is the surface gradient operator given by

$$
\nabla_{s} \theta=\mathbf{P} \nabla \theta
$$

for any differentiable scalar field $\theta$ defined on $\Gamma$. Note that the temperature field $T$ and its surface gradient $\nabla_{s} T$ are well-defined on $\Gamma$ owing to the continuity condition (1) and fact that $\nabla_{s} T=\nabla_{s} T^{(1)}-\nabla_{s} T^{(2)}$ on $\Gamma$.

The temperature field $T$ and heat flux field over $\Omega$ can be expressed in terms 
of $T^{(i)}$ and $\mathbf{q}^{(i)}$ by

$$
T(\mathbf{x})=\sum_{i=1}^{2} \chi^{(i)}(\mathbf{x}) T^{(i)}(\mathbf{x}), \quad \mathbf{q}(\mathbf{x})=\sum_{i=1}^{2} \chi^{(i)}(\mathbf{x}) \mathbf{q}^{(i)}(\mathbf{x})
$$

where $\chi^{(i)}(\mathbf{x})$ is the characteristic function of $\Omega^{(i)}$ such that $\chi^{(i)}(\mathbf{x})=1$ for $\mathbf{x} \in \Omega^{(i)}$ and $\chi^{(i)}(\mathbf{x})=0$ for $\mathbf{x} \notin \Omega^{(i)}$. The boundary conditions on $\partial \Omega$ are in general mixed:

$$
\left\{\begin{array}{l}
\mathbf{q} \cdot \boldsymbol{\nu}=-\bar{q}_{\nu} \text { on } \partial \Omega_{q} \\
T=\bar{T} \text { on } \partial \Omega_{T}
\end{array}\right.
$$

where $\boldsymbol{\nu}$ is the outward unit vector normal to $\partial \Omega, \bar{q}_{\nu}$ and $\bar{T}$ are the heat normal flux and temperature prescribed on $\partial \Omega_{q}$ and $\partial \Omega_{T}$, respectively.

To specify the physical background and the validity domain of the HC interface model used to describe $\Gamma$, let us consider two configurations as shown by Fig. 1. In the configuration (a), the representative volume element $\Omega$ consists of an inclusion $\hat{\Omega}^{(1)}$ inserted in to a matrix $\hat{\Omega}^{(2)}$ via an interphase $\Omega^{(0)}$. The interface between $\hat{\Omega}^{(1)}$ and $\Omega^{(0)}$ and the interface between $\hat{\Omega}^{(2)}$ and $\Omega^{(0)}$ are assumed to be perfect, and the the thickness $h$ of $\Omega^{(0)}$ is required to be uniform and very small in comparison with the typical size, say the radius, of the inclusion. In the configuration (b), the interphase is replaced by an interface of zero thickness located at the middle surface $\Gamma$ of the interphase, and the inclusion and matrix are extended up to the middle surface $\Gamma$, so as to occupy the subdomains $\Omega^{(1)}$ and $\Omega^{(2)}$, respectively. The jump conditions that the imperfect interface $\Gamma$ has to verify for the configurations (a) and (b) to be physically equivalent were derived first by Sanchez-Palencia [22] in a particular case and then by Hashin [10] and Benveniste [4] in the general situation. When the thermal conductivity tensors, $\mathbf{K}^{(1)}, \mathbf{K}^{(2)}$ and $\mathbf{K}^{(0)}$, of the inclusion, matrix and interphase are such that $\left\|\mathbf{K}^{(0)}\right\|>>\left\|\mathbf{K}^{(1)}\right\|$ and $\left\|\mathbf{K}^{(0)}\right\|>>\left\|\mathbf{K}^{(2)}\right\|$, the interphase is said to be HC. In this case, the conditions that the imperfect interface $\Gamma$ must satisfy are those of the $\mathrm{HC}$ interface model detailed above. In particular, the interface thermal conductivity tensor $\mathbf{K}_{s}$ of $\Gamma$ can be expressed in terms of $\mathbf{K}^{(0)}$ and $h$ (see, e.g., [4]):

$$
\mathbf{K}_{s}=h \mathbf{S}^{(0)} \quad \text { with } \quad \mathbf{S}^{(0)}=\mathbf{K}^{(0)}-\frac{\left(\mathbf{K}^{(0)} \mathbf{n}\right) \otimes\left(\mathbf{K}^{(0)} \mathbf{n}\right)}{\mathbf{K}^{(0)}:(\mathbf{n} \otimes \mathbf{n})}
$$

where $\mathbf{n}$ is the unit normal to $\Gamma$. When $\mathbf{K}^{(0)}$ is isotropic, i.e. $\mathbf{K}^{(0)}=k^{(0)} \mathbf{I}$, it follows from (14) that

$$
\mathbf{K}_{s}=k_{s} \mathbf{P}, k_{s}=h k^{(0)}
$$




\subsection{Weak form}

The strong formulation of the thermal conduction in a particulate composite with HC interface is given by (6), (7), (9), (10) and (13). In order to numerically solve this problem, we proceed now to establish the corresponding weak formulation.

Let $\delta T$ be any continuous temperature field over $\Omega$, which is differentiable everywhere except on the interface $\Gamma$ and verifies the boundary condition

$$
\delta T=0 \text { on } \partial \Omega_{T} .
$$

Starting from (6), we can write

$$
\int_{\Omega^{(i)}} \operatorname{div} \mathbf{q}^{(i)} \delta T d \Omega=0
$$

with $i=1$ and 2 . Invoking the divergence theorem, using the fact that $\partial \Omega^{(1)}=$ $\Gamma$ and $\partial \Omega^{(2)}=\partial \Omega \cup \Gamma$, and accounting for (7), we deduce from the above two equations that

$$
\begin{aligned}
& \int_{\Omega^{(1)}} \mathbf{q}^{(1)} \cdot \nabla(\delta T) d \Omega-\int_{\Gamma}\left(\mathbf{q}^{(1)} \cdot \mathbf{n}\right) \delta T d S=0, \\
& \int_{\Omega^{(2)}} \mathbf{q}^{(2)} \cdot \nabla(\delta T) d \Omega+\int_{\Gamma}\left(\mathbf{q}^{(2)} \cdot \mathbf{n}\right) \delta T d S-\int_{\partial \Omega q}\left(\mathbf{q}^{(1)} \cdot \nu\right) \delta T d S=0 .
\end{aligned}
$$

Adding the foregoing two equations yields

$$
\int_{\Omega} \mathbf{q} \cdot \nabla(\delta T) d \Omega+\int_{\Gamma}\left(\mathbf{q}^{(2)}-\mathbf{q}^{(1)}\right) \cdot \mathbf{n} \delta T d S+\int_{\partial \Omega_{q}} \bar{q}_{\nu} \delta T d S=0 .
$$

Next, accounting for (7), the precedent equation becomes

$$
\int_{\Omega} \mathbf{q} \cdot \nabla(\delta T) d \Omega-\int_{\Gamma}\left(\operatorname{div}_{s} \mathbf{q}_{s}\right) \delta T d S+\int_{\partial \Omega_{q}} \bar{q}_{\nu} \delta T d S=0 .
$$

With the help of the identity

$$
\operatorname{div}_{s}(\theta \mathbf{w})=\theta \operatorname{div}_{s} \mathbf{w}+\nabla_{s} \theta \cdot \mathbf{w}
$$

holding for a surface scalar field $\theta$ and a surface vector field $\mathbf{w}$ on $\Gamma$, equation (21) can be further written as

$$
\int_{\Omega} \mathbf{q} \cdot \nabla(\delta T) d \Omega+\int_{\Gamma} \mathbf{q}_{s} \cdot \nabla_{s}(\delta T) d S-\int_{\Gamma} d i v_{s}\left(\delta T \mathbf{q}_{s}\right) d S=-\int_{\partial \Omega_{q}} \bar{q}_{\nu} \delta T d S .
$$


By hypothesis, $\Gamma$ is a closed surface, so that

$$
\int_{\Gamma} \operatorname{div}_{s}\left(\delta T \mathbf{q}_{s}\right) d S=0
$$

by application of the Stokes' theorem. Consequently, equation (21) becomes

$$
\int_{\Omega} \mathbf{q} \cdot \nabla(\delta T) d \Omega+\int_{\Gamma} \mathbf{q}_{s} \cdot \nabla_{s}(\delta T) d S=-\int_{\partial \Omega_{q}} \bar{q}_{\nu} \delta T d S .
$$

This equation holds independently of the bulk and interface conduction laws.

When the bulk and interface Fourier's laws (9) (10) apply, we introduce them into (25) to obtain

$$
\int_{\Omega}\left(\mathbf{K}^{(i)} \nabla T\right) \cdot \nabla(\delta T) d \Omega+\int_{\Gamma}\left(\mathbf{K}_{s} \nabla_{s} T\right) \cdot \nabla_{s}(\delta T) d S=\int_{\partial \Omega_{q}} \bar{q}_{\nu} \delta T d S .
$$

This is the weak formulation needed for the numerical modelling of the problem under consideration. Note that the second term in the left-hand side reflects the HC interface effect.

\section{Discretization of the problem by LSM and XFEM}

\subsection{Interface normal evaluation}

The domain $\Omega$ is discretized by nodes that do not necessarily match the interface $\Gamma$. Here tetrahedra are adopted for meshes, whereas any other elements can be used. Regular meshes can then be taken for parallelepipedic domains, even if the interface is arbitrarily shaped.

The interface $\Gamma$ is defined by (3). Wherever needed, the components of the unit normal vector $\mathbf{n}(\mathbf{x})$ given by (4) can be evaluated by

$$
\tilde{\mathbf{n}}(\mathbf{x})=\frac{\boldsymbol{\nabla} \tilde{\phi}(\mathbf{x})}{\|\boldsymbol{\nabla} \tilde{\phi}(\mathbf{x})\|}
$$

with

$$
\nabla_{i} \tilde{\phi}(\mathbf{x})=\sum_{j=1}^{n} \frac{\partial N_{j}(\mathbf{x})}{\partial x_{i}} \phi_{j},
$$

where $N_{j}(\mathbf{x})$ are the standard finite element shape functions, $\phi_{j}$ are the nodal values of the level-set function $\phi$, and $n$ is the number of nodes of an element. In the present paper, we use simple linear finite element shape functions, though 
higher-order shape functions can be employed [3]. The above approximation for $\mathbf{n}$ can then be used to evaluate the components of the projector $\mathbf{P}$ defined by (5) and involved in the weak form (26).

\subsection{Discretisation}

In the highly conducting interface model, the temperature must be continuous at the interface whereas the normal component of its gradient to the interface is in general discontinuous and the tangential components are continuous. These conditions are now enforced by adding to the standard finite element field an enrichment term as suggested by the XFEM method $[20,2]$. Thus, the approximation at a point $\mathbf{x}$ lying in an element $\Omega_{e}$ becomes

$$
T^{h}(\mathbf{x})=\sum_{i=1}^{n} N_{i}(\mathbf{x}) T_{i}+\sum_{j=1}^{m} N_{j}(\mathbf{x}) \psi(\mathbf{x}) b_{j}
$$

In the second term of the right side of this expression, $N_{j}(\mathbf{x})$ are the shape functions of the nodes of an element whose support is cut by the interface and $\psi(\mathbf{x})$ is a function verifying the discontinuity/continuity at the interface. In Eq. (29), $T_{i}$ and $b_{j}$ are the coefficients related to enriched elements cut by the interface. In non enriched elements, $T_{i}$ represent the nodal temperatures. More details can be found for example in [20,3,23,25], among many papers on XFEM.

From the numerical point of view, the specificity of the present problem is the presence of the internal thermal energy term in Eq. (26) related to the interface. To compute the associated surface integral, we first approximate the interface by triangular facets. For this purpose, we seek for the intersection between the level-set function $\phi(\mathbf{x})$ and the tetrahedral mesh. The tetrahedra cut by the interface $\Gamma$ can be easily detected, as the values of $\phi$ evaluated at the two nodes of a given edge have opposite signs. The intersection between $\phi(\mathbf{x})=$ 0 and the edges of the mesh are then approximated by a linear interpolation of $\phi(\mathbf{x})$ based on the mesh. A Gauss integration is then performed on each triangular facet of the interface approximation. In this work, four Gauss points are used in the tetrahedra cut by the interface, one in the remaining tetrahedra and three on each triangular facet of the approximated interface.

Substituting the trial and test functions from Eq. (29) into Eq. (26), and using the arbitrariness of nodal variations, the following discrete system of linear equations is obtained:

$$
\left(\hat{\mathbf{K}}+\hat{\mathbf{K}}_{s}\right) \mathbf{T}=\hat{\mathbf{q}}
$$


where $\hat{\mathbf{K}}$ and $\hat{\mathbf{K}}_{s}$ are the bulk and surface impedance matrices, respectively, $\hat{\mathbf{q}}$ is the vector of external flux and $\mathbf{T}$ is a vector containing the unknowns associated to classical and enrichment degrees of freedom, according to Eq. (29). More precisely, the matrices $\hat{\mathbf{K}}$ and $\hat{\mathbf{K}}_{s}$ and vector $\hat{\mathbf{q}}$ are defined by

$$
\begin{aligned}
& \hat{\mathbf{K}}_{I J}=\int_{\Omega} \mathbf{B}_{I}^{T} \mathbf{K}_{i}^{(i)} \mathbf{B}_{J} d \Omega, \\
& \hat{\mathbf{K}}_{s I J}=\int_{\Gamma} \mathbf{B}_{I}^{T} \mathbf{P} \mathbf{K}_{s} \mathbf{P} \mathbf{B}_{J} d S, \\
& \hat{\mathbf{q}}=\int_{\partial \Omega_{q}} \mathbf{N}^{T} \bar{q}_{n} d S,
\end{aligned}
$$

where $\mathbf{B}$ and $\mathbf{N}$ are the matrices of shape functions derivatives and shape functions associated with the approximation scheme (29). More details about practical implementation can be found e.g. in $[20,3,23,25]$.

\section{Numerical examples and discussion}

\subsection{Convergence analysis}

We consider a spherical inclusion $\Omega^{(1)}$ of radius $r_{1}$ coated with a spherical shell $\Omega^{(2)}$ of exterior radius $r_{2}$. This spherical composite is submitted to a homogeneous temperature gradient field

$$
T=\bar{T}=-\mathbf{e}_{0} \cdot \mathbf{x}
$$

on its boundary $\partial \Omega$ with $\mathbf{e}_{0}$ being a constant vector. Throughout this paper, we will assume that the bulk materials are isotropic, i.e. $\mathbf{K}^{(i)}=k_{i} \mathbf{1}$ and that the imperfect interface is also isotropic in the tangent plane to the interface, i.e. $\mathbf{K}_{s}=k_{s} \mathbf{P}$. The analytical exact solution of the temperature field for this problem was provided in [15] and is recalled in Appendix 1 to make the paper self-contained. To test our numerical procedure, we compare the obtained numerical solution and the exact one. A cubic box containing the inclusion but included in the spherical shell is cut of the composite sphere. The exact solution of the problem is then prescribed on the boundary of the cubic domain. The cubic domain is meshed with tetrahedra. The spherical inclusion is defined implicitly by a level-set function which takes negative values inside the spherical inclusion, and positive values outside the latter (see e.g. [23]). A convergence analysis is carried out, using different meshes with increasing nodal densities associated with a characteristic element size $h$. For each mesh, we compute the relative energy error norm defined by: 


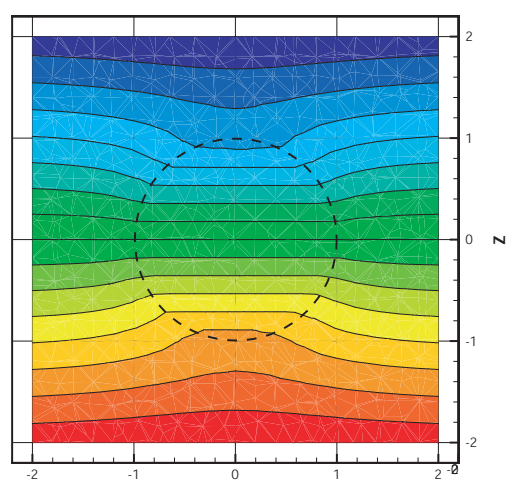

(a)

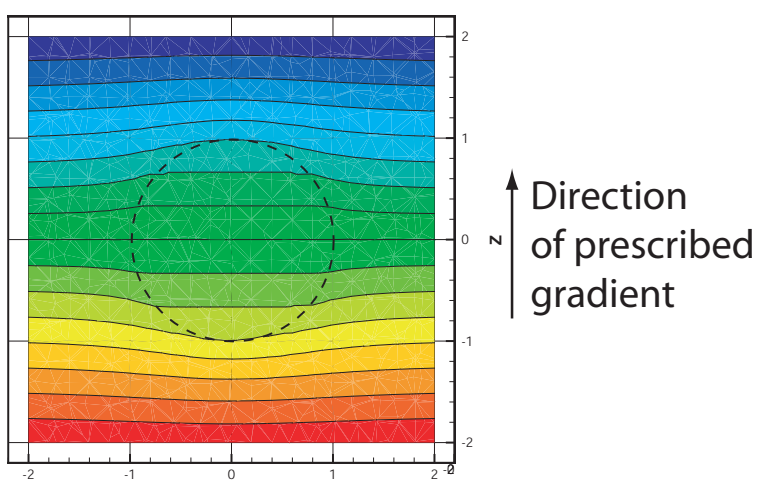

(b)

Fig. 2. Effects of the imperfect interface on the temperature field near the inclusion: (a) $k_{s}=0 \mathrm{~W} \cdot \mathrm{K}^{-1}$; (b) $k_{s}=10^{-6} \mathrm{~W} \cdot \mathrm{K}^{-1}$.

$$
\begin{gathered}
\frac{\left\|T^{h}(\mathbf{x})-T(\mathbf{x})\right\|_{E(\Omega)}}{\|T(\mathbf{x})\|_{E(\Omega)}}= \\
\frac{\left\{\int_{\Omega}\left[\boldsymbol{\nabla} T^{h}(\mathbf{x})-\boldsymbol{\nabla} T(\mathbf{x})\right]^{T} \mathbf{K}^{(i)}(\mathbf{x})\left[\boldsymbol{\nabla} T^{h}(\mathbf{x})-\boldsymbol{\nabla} T(\mathbf{x})\right]\right\}^{\frac{1}{2}}}{\left[\int_{\Omega} \boldsymbol{\nabla} T(\mathbf{x})^{T} \mathbf{K}^{(i)}(\mathbf{x}) \boldsymbol{\nabla} T(\mathbf{x})\right]^{\frac{1}{2}}},
\end{gathered}
$$

where $(.)^{h}(\mathbf{x})$ and $().(\mathbf{x})$ denote the approximated and exact solutions, respectively. The following numerical parameters are used (see Appendix 1): $r_{1}=1$ $\mu \mathrm{m}, r_{2}=3 \mu \mathrm{m}, k_{1}=1 \mathrm{~W} \cdot \mathrm{m}^{-1} \cdot \mathrm{K}^{-1}, k_{2}=1 \mathrm{~W} \cdot \mathrm{m}^{-1} \cdot \mathrm{K}^{-1}$ and $\mathbf{e}_{0}=(0,0,1)^{T}$. We study the convergence of the numerical solution for different values of the surface conductivity $k_{s}$, ranging from $k_{s}=0 \mathrm{~W} . \mathrm{K}^{-1}$ to $k_{s}=10^{-6} \mathrm{~W} . \mathrm{K}^{-1}$. In Fig. 2, the temperature field is depicted in a plane passing through the origin of the inclusion and being parallel to the direction of the prescribed temperature gradient $\mathbf{e}_{0}$. We can observe the effect of the $\mathrm{HC}$ imperfect interface on the temperature field.

The convergence results are plotted in Fig. 3. We note that the numerical solution converges to the exact solution, with the rate close to the optimal value which is equal to 1 in terms of the energy error norm for linear finite elements. The convergence rate remains unchanged for different values of the surface conductivity $k_{s}$. The accuracy of the proposed numerical approach is thus exampled. 


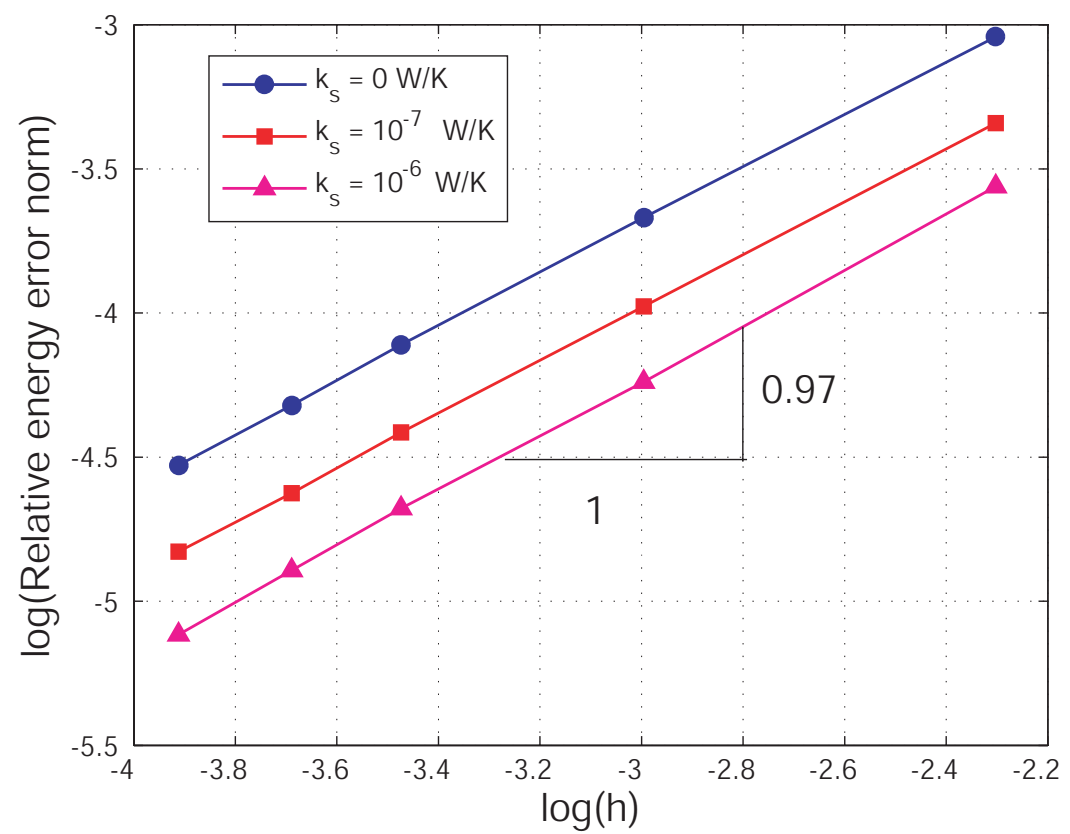

Fig. 3. Convergence analysis of the computed solution with respect to the analytical solution, for different surface conductivities.

\subsection{Homogenization of a composite containing spheroidal inclusions with highly conducting interfaces}

In this example we aim at computing the effective conductive properties of a periodic heterogeneous material containing spheroidal inhomogeneities with highly conducting imperfect interfaces. We discretize a representative volume element using a mesh of tetrahedra, which is constructed independently of the interface geometry. As in [23], we model a spheroidal interface by using an appropriate level-set function. Examples of intersections between the mesh and the level-set function for spheroidal inhomogeneities are depicted in Fig. 4 .

We consider aligned spheroidal inclusions whose semi-axes are parallel to the axes of $x, y$, and $z$ of the global reference frame. The lengths $a_{1}, a_{2}$ and $a_{3}$ of the semi-axes along $x, y$ and $z$ of the spheroid, and the lengths of the enclosing box sides $L_{1}, L_{2}$ and $L_{3}$ vary in the same ratio. We compute numerically the effective conductivities of the composite by applying uniform heat flux at the boundary (see e.g. [7] for more details). To test the accuracy of our procedure, we compare our numerical solution with the analytical estimate for homogenized conductive properties of materials containing ellipsoidal inhomogeneities with highly conducting interface provided by Duan et al. in [6]. Use is made of the following numerical parameters: $k_{1}=0.1 \mathrm{~W} \cdot \mathrm{m}^{-1} \cdot \mathrm{K}^{-1}$, $k_{2}=1 \mathrm{~W} \cdot \mathrm{m}^{-1} \cdot \mathrm{K}^{-1}, a_{1}=1 \mu \mathrm{m}, k_{s}=0 \mathrm{~W} \cdot \mathrm{K}^{-1}, k_{s}=10^{-7} \mathrm{~W} \cdot \mathrm{K}^{-1}$, and the 

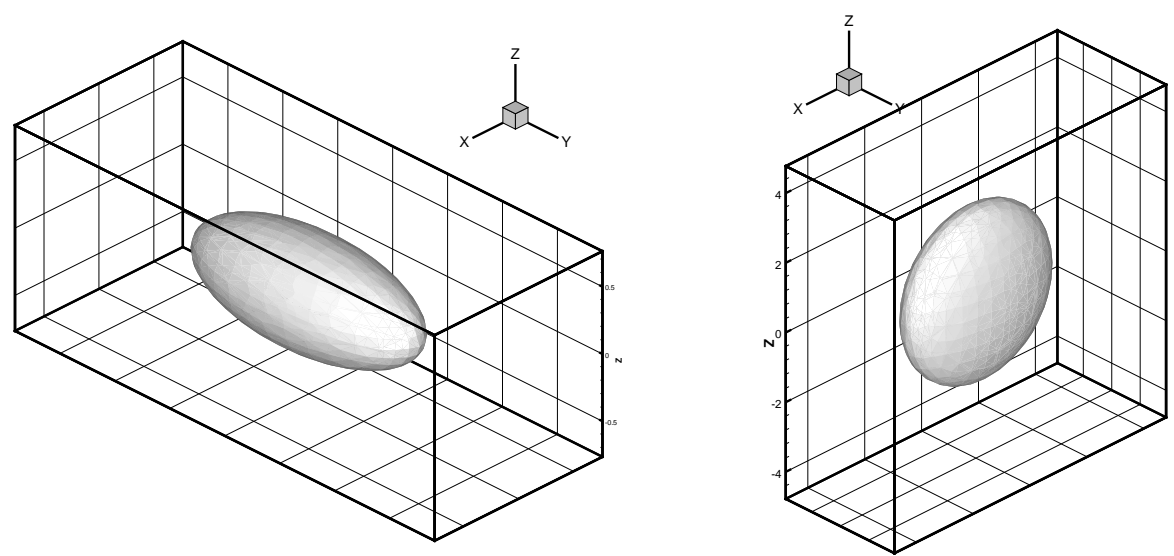

Fig. 4. Examples of spheroidal inhomogeneities modeled by the level-set method through regular meshes: (a) $a_{1}=1, a_{2}=a_{3}=0.4$; (b) $a_{1}=1, a_{2}=a_{3}=2.5$; $f=0.1$.

inclusion fraction $f$ is equal to 0.1. A mesh composed of 44552 tetrahedra (roughly $21 \times 21 \times 21$ nodes) is constructed. In Figs. 5-6, we compare the effective transverse and longitudinal conductivities with respect to the size aspect $\gamma=a_{1} / a_{2}=a_{1} / a_{3}$. The effective conductivity is normalized with respect to $k_{0}=k^{e f f}\left(\gamma=1, k_{s}=0\right)$. The Figs. 5 and 6 show good agreement between the present numerical solution and the estimation derived by Duan et al. [6]. In the case of extreme values of the aspect ratio $\gamma$, which correspond to either very flat or very elongated spheroids, the difference between them becomes slightly accentuated. This can be explained by the fact that, when the inclusion has a high aspect ratio, the preservation of the same accuracy necessitates refining the mesh.

\subsection{Size-dependent effective thermal conductivities of a material containing inclusions with highly conducting interfaces}

Due to the presence of a surface energy term in the weak form of the balance equations (26), and owing to the non constant surface to volume ratio when the size of the inclusion vary, the effective properties of materials containing imperfect interfaces are size-dependent. To study this effect, we consider a cubic representative volume element with a spherical inclusion coated by a much more conducting material than the media constituting the inclusion or the matrix. We then vary the radius $r_{1}$ of the inclusion and the length $L$ of the edges of the cube so as to preserve the inclusion volume fraction. The conductivity of both matrix and inclusion is $k_{1}=k_{2}=0.1 \mathrm{~W} \cdot \mathrm{m}^{-1} \cdot \mathrm{K}^{-1}$. The conductivity of the material is $k_{0}=100 \mathrm{~W} \cdot \mathrm{m}^{-1} \cdot \mathrm{K}^{-1}$ and the interphase between inclusion and matrix is assumed to have a thickness $h=10 \mathrm{~nm}$. We then replace the interphase problem by an inclusion problem with an imperfect 


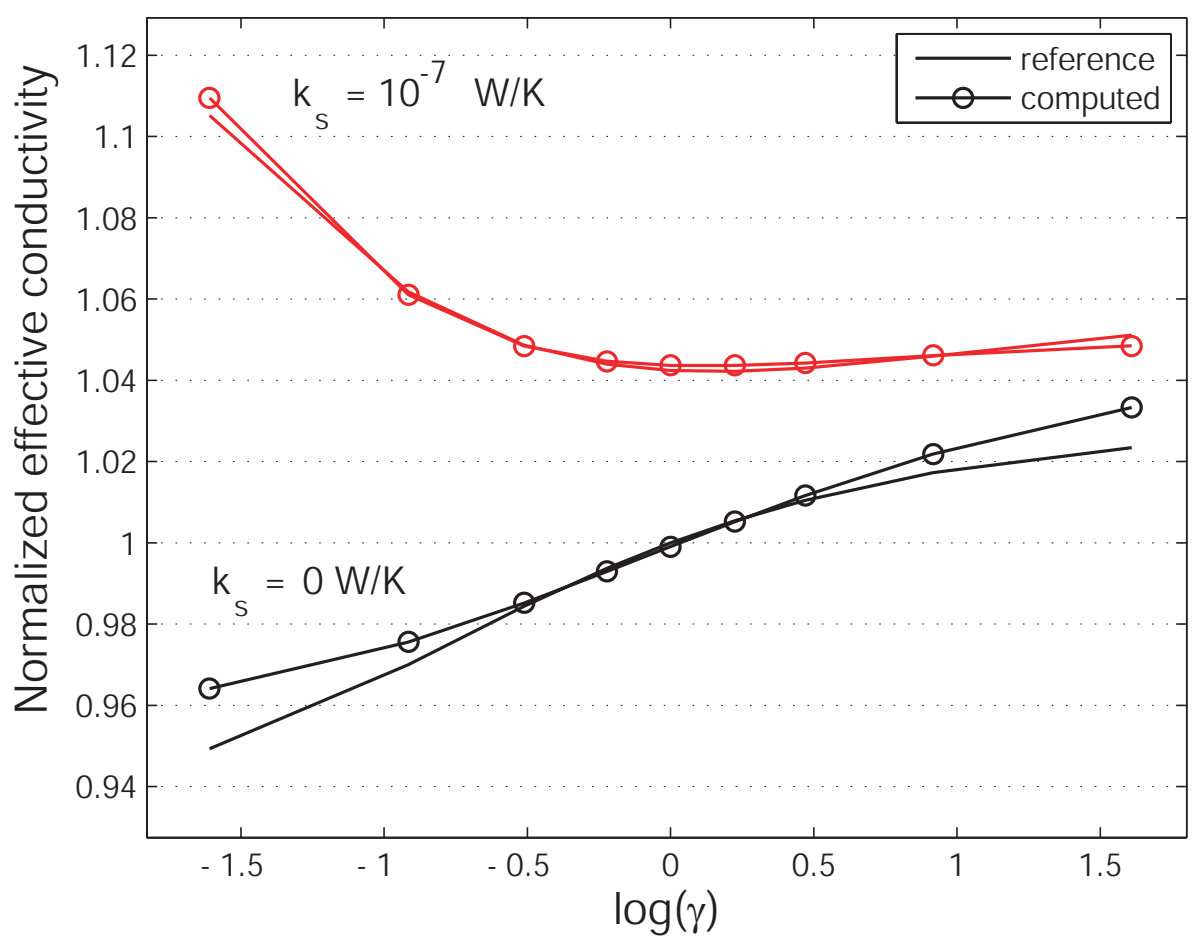

Fig. 5. Effective transverse conductivity of a material containing spheroidal inclusions with imperfect interface with respect to the aspect ratio $\gamma$.

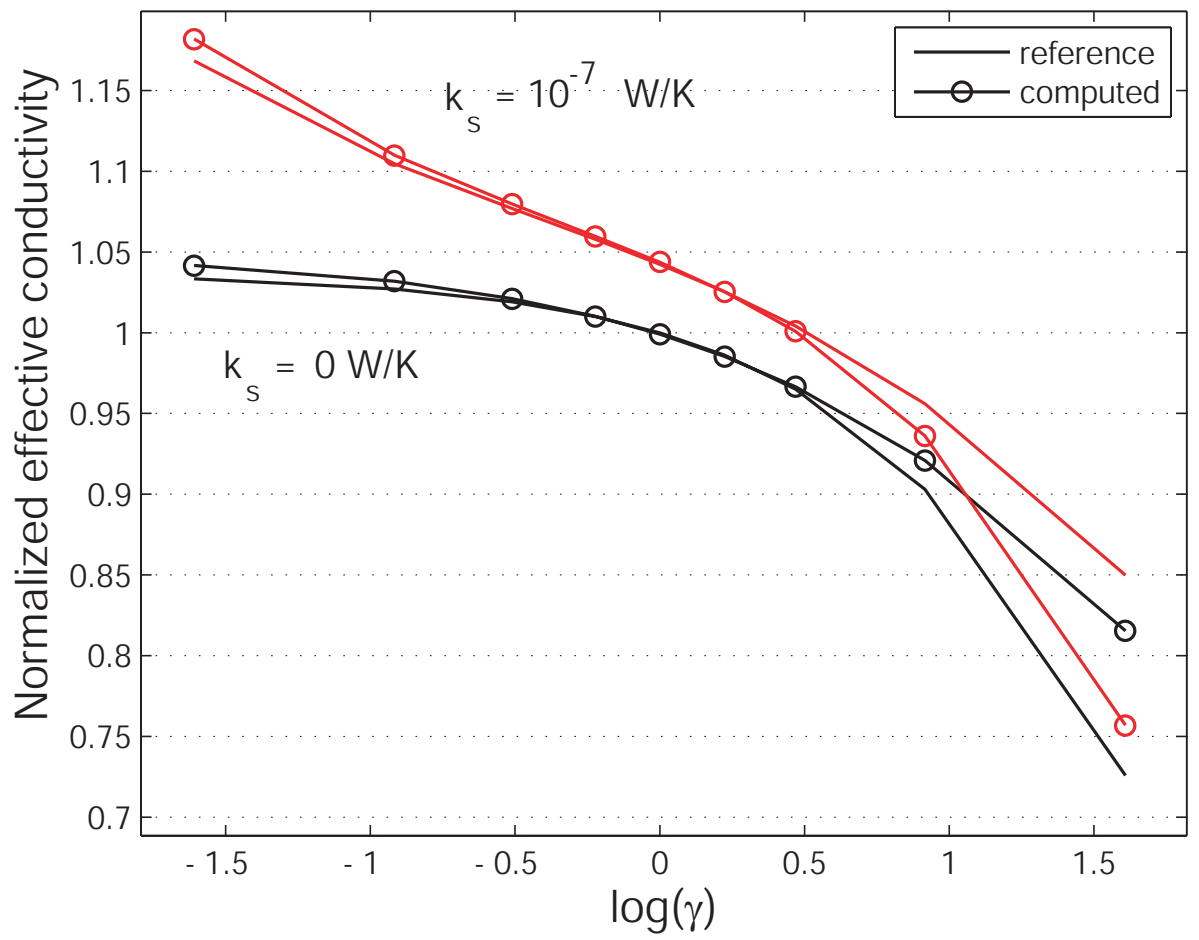

Fig. 6. Effective longitudinal conductivity of the material containing spheroidal inclusions with imperfect interface with respect to the aspect ratio $\gamma$. 


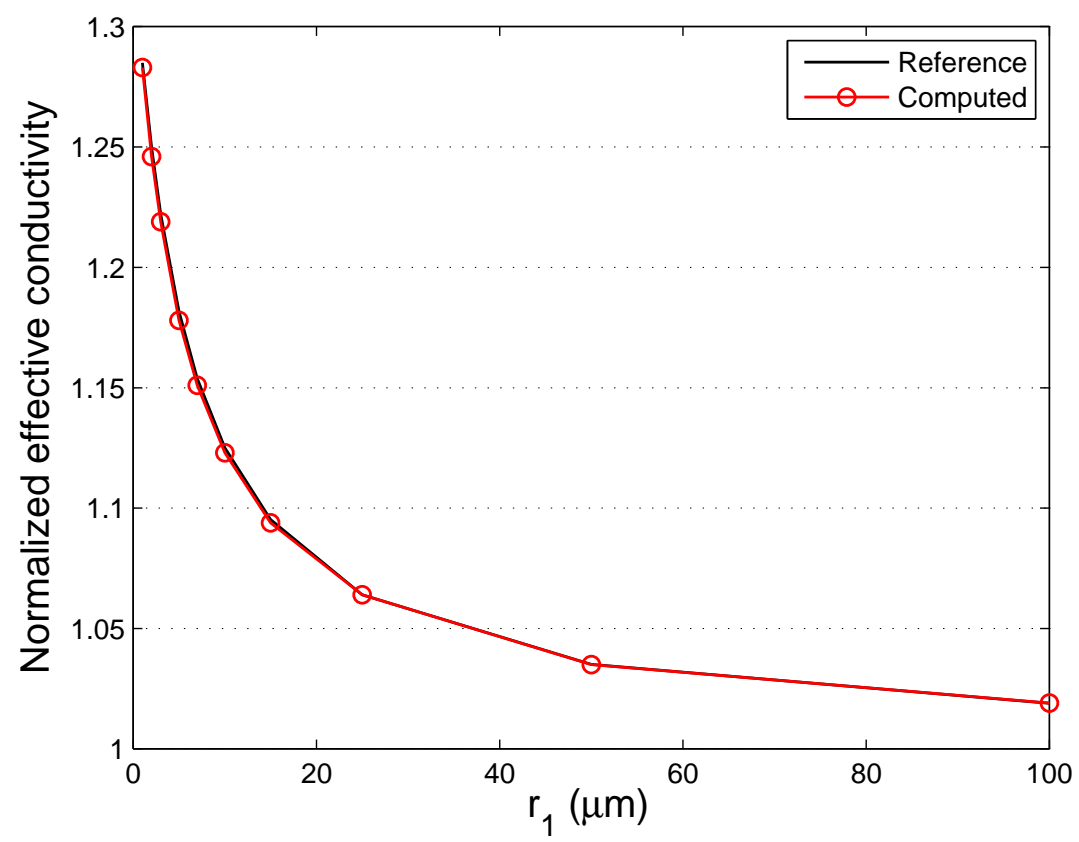

Fig. 7. Size-dependent effective conductivity of a material containing coated microscopic inclusions with highly conducting interface, $f=0.1$

$\mathrm{HC}$ interface. The resulting surface parameter $k_{s}$ is thus given by $k_{s}=10^{-6}$ W.K $\mathrm{K}^{-1}$ according to Eq. (15).

A mesh composed of 44552 tetrahedra (roughly $21 \times 21 \times 21$ nodes) is employed. We compare in Figs. 7 and 8 the numerical results obtained through the proposed numerical procedure and the estimation given by Le Quang et al. in [15] using a generalized self-consistent model proposed. We note that the proposed methodology is in excellent agreement with the estimation and is able to capture the size-effects accurately. It is shown that smaller the inclusions are, the higher are the effective properties.

In figure 9 we compute the normalized effective conductivity of the same material for different inclusion volume fractions and radii $r_{1}$. The size effects are here again clearly visible.

\subsection{Shape effects}

One of the main advantages of the proposed numerical framework is its ability to treat arbitrarily shaped inclusions. Now let us take this advantage to investigate the influence of the inclusion shape on the effective conductive properties of a composite with a HC interface. For this purpose, we propose 


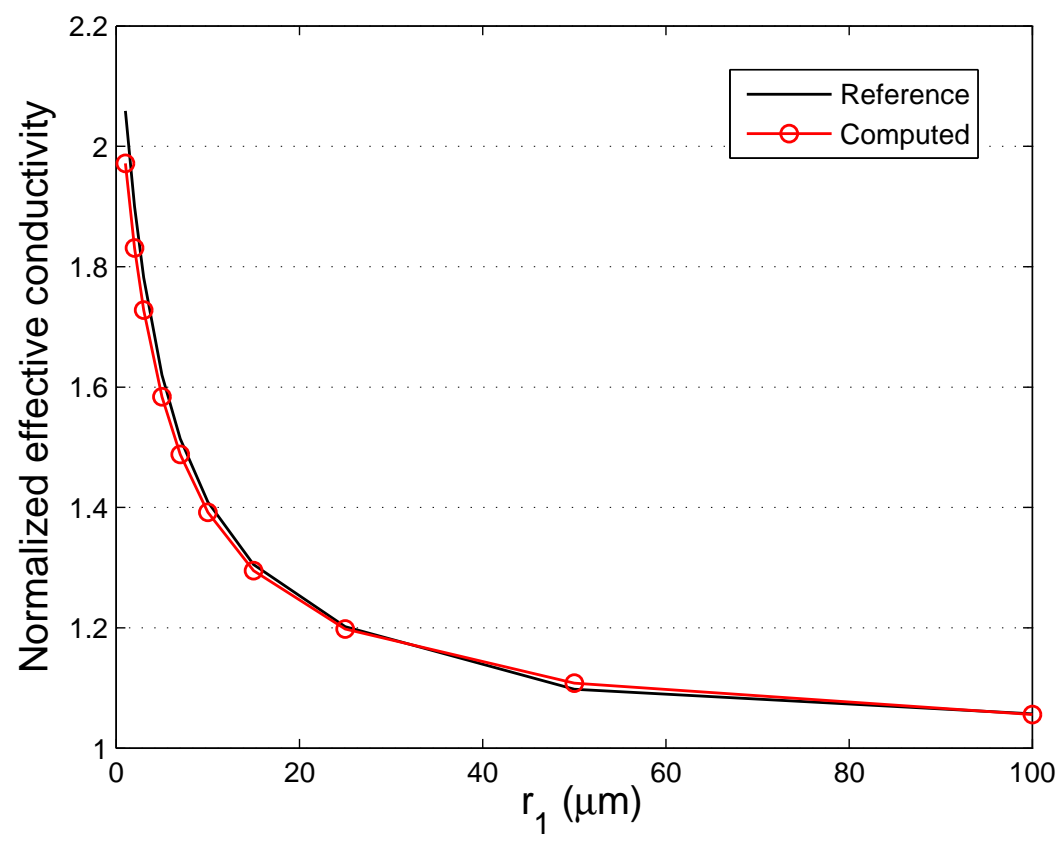

Fig. 8. Size-dependent effective conductivity of a material containing coated microscopic inclusions with highly conducting interface, $f=0.3$

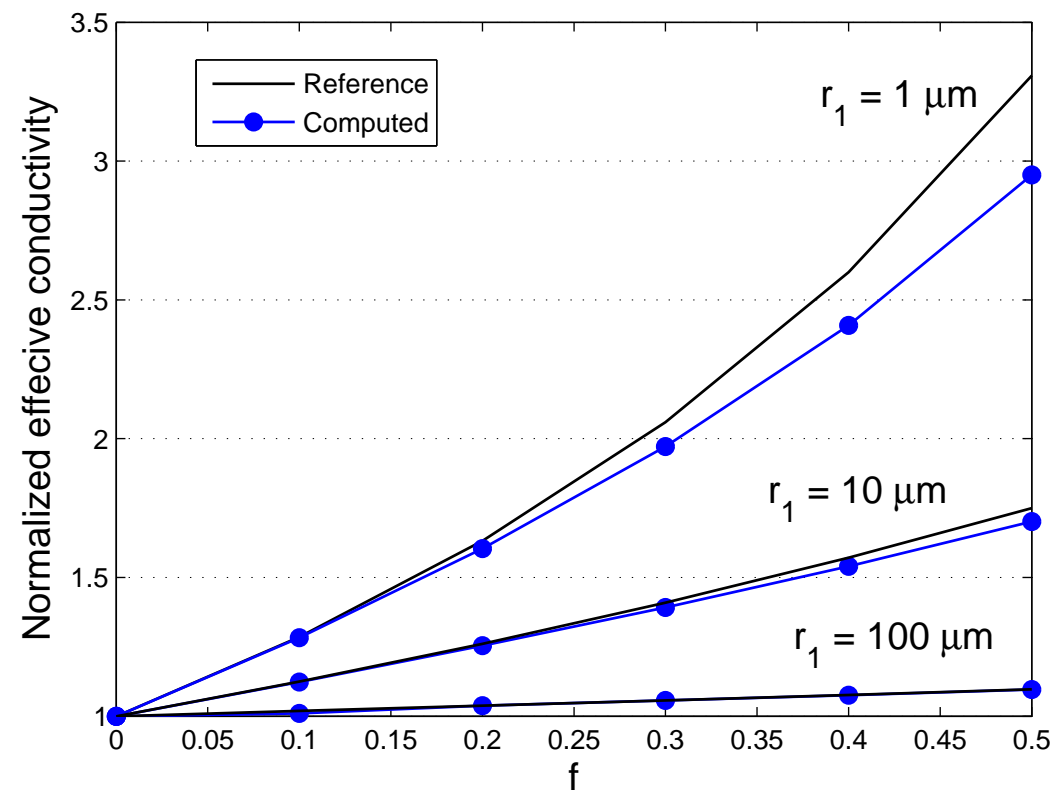

Fig. 9. Effective conductivity of a material containing coated microscopic inclusions with highly conducting interface for different volume fractions and inclusion radii. 

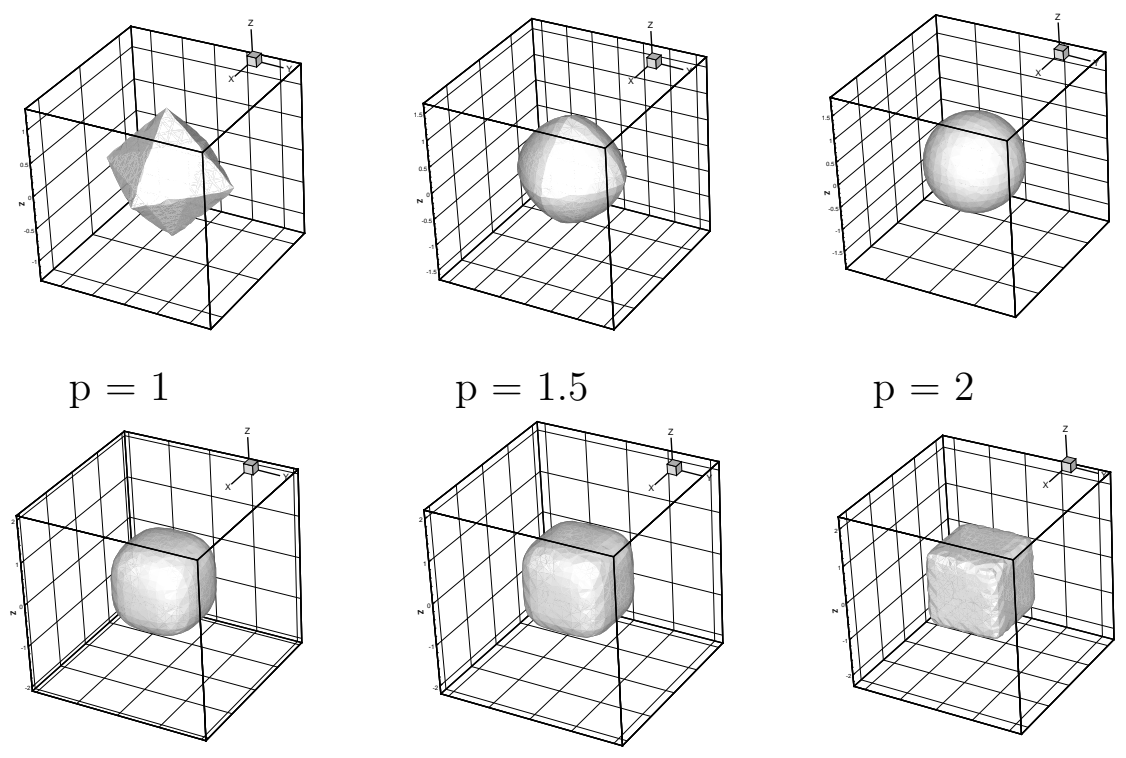

$\mathrm{p}=3$

$\mathrm{p}=4$

$\mathrm{p}=10$

Fig. 10. Inclusions of different shapes and with the same volume fraction $f=0.1$.

the following versatile level-set function

$$
\phi(\mathbf{x})=\left(\frac{\left|x-x_{c}\right|}{a_{1}}\right)^{p}+\left(\frac{\left|y-y_{c}\right|}{a_{2}}\right)^{p}+\left(\frac{\left|z-z_{c}\right|}{a_{3}}\right)^{p}-1
$$

where $x_{c}, y_{c}$ and $z_{c}$ denote the coordinates of the inclusion centre, to describe a rich class of inclusions in a uniform way. Different examples of inclusions defined by this level-set function are depicted in Fig. 10. For $p=1$, the inclusion is an octahedron, for $p=2$ the inclusion is an ellipsoid and for $p \rightarrow \infty$ the shape is a parallelepiped. By varying continuously $p$, other complex shapes can be generated.

Let $L_{i}$ the edges lengths of the enclosing parallelepipedic box. We assume that $L_{i}=2 \beta a_{i}$. When $a_{i}$ are fixed, a representative volume element with a given volume fraction $f$ can be defined by computing:

$$
\beta=\frac{1}{2}\left(\frac{V_{i n c}}{f a_{1} a_{2} a_{3}}\right)^{\frac{1}{3}}
$$

The volume $V_{i n c}$ of such an inclusion occupying the center of a representative volume element (Fig. 10) is determined numerically in the present work.

Use is made of the following numerical parameters: $k_{1}=0.01 \mathrm{~W} \cdot \mathrm{m}^{-1} \cdot \mathrm{K}^{-1}$, $k_{2}=1 \mathrm{~W} \cdot \mathrm{m}^{-1} \cdot \mathrm{K}^{-1}$. The numerical results are provided in Figs. 11-12 for varying shape factor $p$ and for two volume fractions $f=0.1, f=0.3$. For each shape, the inclusion volume fraction is identical and the values of $a_{1}=a_{2}=a_{3}$ in Eq. (36) are fixed to $1 \mu \mathrm{m}$. Here again, we model inclusions coated with 


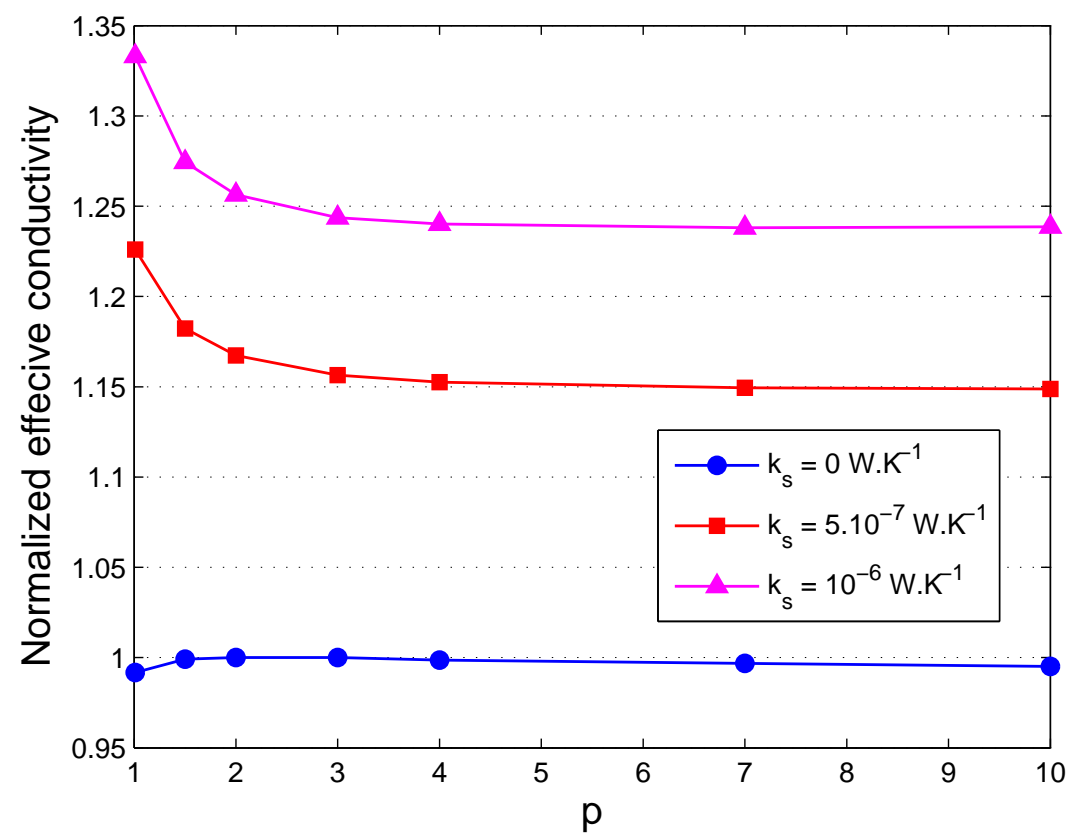

Fig. 11. Effects of shape and surface conductivity on the effective conductivity of a composite, $f=0.1$.

a highly conducting material. The thickness of the coating is assumed to be $10 \mathrm{~nm}$, and two conductivities are tested for the coating material: $k^{(0)}=50$ $W \cdot m^{-1} \cdot K^{-1}$ and $k^{(0)}=100 W \cdot m^{-1} \cdot K^{-1}$. We then replace the inclusion problem by an inclusion problem with an imperfect $\mathrm{HC}$ interface. The resulting surface parameters are $k_{s}=5 \cdot 10^{-7} \mathrm{~W} . \mathrm{K}^{-1}$ and $k_{s}=10^{-6} \mathrm{~W} . \mathrm{K}^{-1}$. The effective conductivity of the composite (which is thermally isotropic owing to the symmetry of the RVE used) is normalized with respect to $k_{0}=k^{e f f}\left(p=2, k_{s}=0\right)$. We note that when no surface effects are considered $\left(k_{s}=0 \mathrm{~W} \cdot \mathrm{K}^{-1}\right)$, the shape dependence is negligible. In contrast, when surface effects occur, the effective conductivity is strongly shape-dependent. Indeed, higher effective conductivities than the ones obtained with a spherical shape can be obtained for particular shapes of inclusions (see Figs. 11 and 12).

\section{Conclusion}

In this work, a weak formulation has been established which is suitable for the numerical computation of the effective thermal conductivities of a particulate composite in which the inclusions have different sizes and arbitrary shapes and the interfaces are highly conducting. An extended finite element method has then been used in tandem with a level-set technique to elaborate an efficient numerical procedure for modelling highly conducting curved interfaces without resort to curvilinear coordinates and surface elements. The 


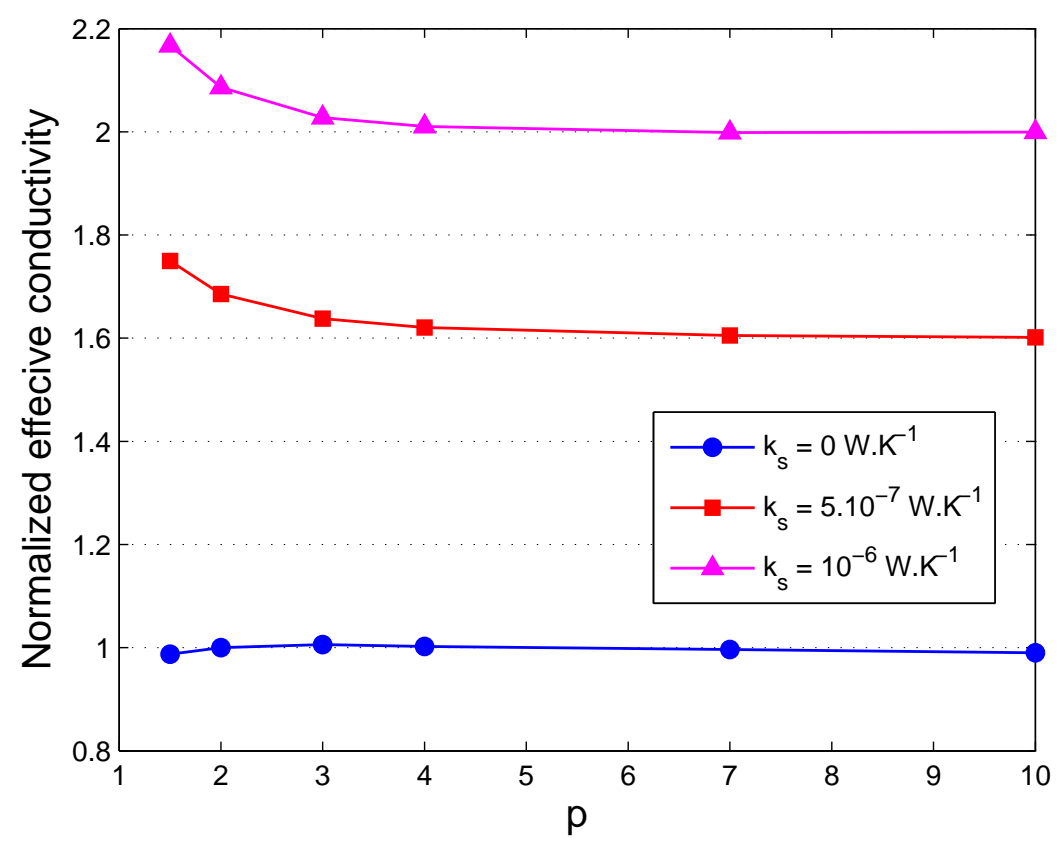

Fig. 12. Effects of shape and surface conductivity on the effective conductivity, $f=0.3$.

elaborated 3D numerical procedure, tested and validated by means of relevant analytical exact and approximate results as benchmarks, has been applied to quantify the size and shape effects on the effective thermal conductivities of particular composites where the inclusions are coated by a highly conducting interface. In particular, it has been found that when no surface effects are considered, the effective conductivity of the material is almost shape independent. In contrast, when surface effects occur, the effective conductivity is strongly shape-dependent. Indeed, higher effective conductivities than the ones obtained with a spherical shape can be obtained for particular shapes of inclusions. The proposed 3D numerical procedure can be applied to computing the effective conductivities of heterogeneous materials with random microstructures and will be helpful for us to extend our previous work [25] to 3D elastic heterogeneous materials with elastic coherent interfaces.

\section{Aknowledgments}

The support this work enjoys from EDF is gratefully acknowledged. The authors wish to thank an anonymous referee for the constructive comments which have allowed us to improve the paper. 


\section{Appendix : Exact solution of the temperature field in a compos- ite sphere with a highly conducting imperfect interface}

A composite sphere of external radius $r_{2}$ composed of a coating and a core of radius $r_{1}$, are separated by a highly conducting interface of conductivity $k_{s}$. This spherical composite is submitted to a homogeneous temperature gradient field

$$
T=\bar{T}=-\mathbf{e}_{0} \cdot \mathbf{x}
$$

on its boundary $\partial \Omega$ with $\mathbf{e}_{0}$ being a constant vector, and $\mathbf{e}^{0}=\left(0,0, e^{0}\right)^{T}$. Both the core and coating forming the composite sphere are assumed to be isotropic and homogeneous. Then the expressions of the temperature and flux field are given in the system of spherical coordinates $(r, \theta, \phi)$ corresponding to a spherical orthogonal basis $\left(\mathbf{e}_{r}, \mathbf{e}_{\theta}, \mathbf{e}_{\phi}\right)$, by (see, e.g. [12])

$$
\begin{aligned}
& T^{(i)}=-\left(a_{i} r+\frac{b_{i}}{r^{2}}\right) \cos \omega \\
& \nabla_{r}^{(i)} T=-\left(a_{i}-2 \frac{b_{i}}{r^{3}}\right) \cos \omega, \nabla_{\theta}^{(i)} T=\left(a_{i}+\frac{b_{i}}{r^{3}}\right) \sin \omega, \nabla_{\phi}^{(i)} T=0 .
\end{aligned}
$$

where $i=1$ and $i=2$ refer to the core and the coating and in which $\omega$ denote the angle between $\mathbf{e}^{0}$ and $\mathbf{e}_{r}$. The heat flux is obtained by $q_{j}^{(i)}=-k_{i} \nabla_{j}^{(i)} T$. By writing the different continuity conditions at $r=r_{1}, r=r_{2}$ and avoiding the singularity at $r=0$, it follows that $b_{1}=0$ and the remaining constants can be determined by solving the following system of equations ([15]):

$$
\begin{aligned}
& a_{1}=a_{2}+\frac{b_{2}}{r_{1}^{3}} \\
& k_{2}\left(a_{2}-2 \frac{b_{2}}{r_{1}^{3}}\right)-k_{1} a_{1}=2 \hat{k}_{s}\left(a_{2}+\frac{b_{2}}{r_{1}^{3}}\right) \\
& a_{2}+\frac{b_{2}}{r_{2}^{3}}=e^{0}
\end{aligned}
$$

where $\hat{k}_{s}=k_{s} / r_{1}, k_{1}$ and $k_{2}$ denote the core and coating conductivities, respectively. 


\section{References}

[1] Balandin AA, Laearenko OL (2003) Mechanism for thermoelectric figure-ofmerit enhancement in regimented quantum dot superlattices, Appl. Phys. Lett. $82(3): 415-417$.

[2] Belytschko T, Black T (1999) Elastic crack growth in finite elements with minimal remeshing, Int. J. Numer. Methods Eng. 45(5):601-620.

[3] Belytchko T, Parimi C, Moës N, Sukumar N, Usui S (2003) Structured extended finite element method for solids defined by implicit surfaces, Int. J. Numer. Methods Eng. 56:609-635.

[4] Benveniste Y (2006) A general interface model for a three-dimensional curved thin anisotropic interphase between two anisotropic media, J. Mech. Phys. Solids 54:708-734.

[5] Cheng H, Torquato S (1997) Effective conductivity of dispersions of spheres with a superconducting interface, Proc. R. Soc. London, Ser. A 453:1331-1344.

[6] Duan HL, Karihaloo BL (2007) Effective thermal conductivities of heterogeneous media containing multiple imperfectly bonded inclusions, Phys. Rev. B 75:064206.

[7] Kanit K, Forest S, Galliet I, Mounoury V, Jeulin D (2003) Determination of the size of the representative volume element for random composites: statistical and numerical approach, Int. J. Solids Struct. 40:3647-3679.

[8] Gurtin ME, Murdoch AI (1975) Continuum theory of elastic-material surfaces, Arch. Ration. Mech. Anal. 57(4):291-323.

[9] Garret KW, Rosenberg HM (1974) The thermal conductivity of epoxy-resin powder composite materials, Materials. J. Phys. D 7:1247-1258.

[10] Hashin Z (2001) Thin interphase/imperfect interface in conduction, J. Appl. Phys. 89:2261-2267.

[11] Hasselman DPH, Donaldson KY (1992) Effect of reinforcement particle size on the thermal conductivity of a particulate-silicon carbide-reinforced aluminum matrix composite, J. Am. Ceram. Soc. 75:3137-3140.

[12] Hervé E (2002) Thermal and thermoelastic behaviour of mutliply coated inclusion-reinforced composites, Int. J. Solids Struct. 39:1041-1058.

[13] Jing GY, Duan HL, Sun XM, Zhang ZS, Xu J, Li YD, Wang JX, Yu DP (2006) Surface effects properties of silver nanowires: contact atomic-force microscopy, Phys. Rev. B. 73(23):235409.

[14] Khitun A, Balandin AA, Liu JL, Wang KL (2000) In-plane lattice thermal conductivity of a quantum-dot superlattice, J. Appl. Phys. 88(2):696-699.

[15] Le Quang H., Bonnet G., He Q-C, Size-dependent Eshelby's tensor fields and effective thermal behavior of nano-inhomogeneities, submitted. 
[16] Lipton R (1997) Variational methods, bounds, and size effects for composites with highly conducting interface, J. Mech. Phys. Solids 45:361-384.

[17] Lipton R, Talbot DRS (2001) Bounds for the effective conductivity of a composite with an imperfect interface, Proc. R. Soc. Lond. A 457:1501-1517.

[18] Miller RE, Shenevoy VB (2000) Size-dependent elastic properties of nanosized structural elements, Nanotechnology. 11(3):139-147.

[19] Miloh T, Benveniste Y (1999) On the effective conductivity of composites with ellipsoidal inhomogeneities and highly conducting interfaces, Proc. R. Soc. London, Ser. A 455:2687-2706.

[20] Moës N, Dolbow J, Belytschko T (1999) A finite element method for crack growth without remeshing, Int. J. Numer. Methods Eng. 46(1):131-156.

[21] Pham Huy H, Sanchez-Palancia E (1974) Phénomènes de transmission à travers des couches minces de conductivité élevée, J. Math. Anal. Appl. 47:284-309.

[22] Sanchez-Palancia E (1970) Comportement limite d'un problème de transmission à travers une plaque faiblement conductice, C. R. Acad. Sci. Paris Ser. A 270:1026-1028.

[23] Sukumar N, Chopp DL, Moës N, Belytschko T (2001) Modeling holes and inclusions by level sets in the extended finite-element method, Comput. Meth. Appl. Mech. Eng. 190:6183-6200.

[24] Torquato S, Rintoul MD (1995) Effect of the interface on the properties of composite media, Phys. Rev. Lett. 75(22):4067-4070.

[25] Yvonnet J, Le Quang H, He Q-C (2007) An XFEM/level set approach to modelling surface/interface effects and to computing the size-dependent effective properties of nanocomposites, Comput. Mech, 42:119-131. 